\title{
Efecto de la actividad antrópica sobre la composición y diversidad de macroinvertebrados acuáticos en el río Cofre (sistema lótico andino colombiano)
}

\section{Effect of activity antrhopic on the composition and diversity of aquatic macroinvertebrate in the Cofre River (Colombian Andean lotic system)}

\author{
Jhonatan Gutiérrez-Garaviz¹, Hilldier Zamora González ${ }^{1}$, Camilo E. Andrade-Sossa ${ }^{1,2}$
}

\begin{abstract}
Resumen
Objetivos: Se analizó el efecto de la actividad antrópica sobre la comunidad de macroinvertebrados acuáticos en el río Cofre, a partir de su composición y diversidad, teniendo en cuenta la variación a nivel espacial y temporal. Metodología: Se definieron cuatro estaciones de muestreo, con base en los cambios registrados en $\mathrm{pH}$ y conductividad; los organismos se colectaron de acuerdo con la metodología propuesta en la presente investigación, acompañados con el registro de algunos parámetros físico-químicos como datos complementarios; para el análisis de información se determinaron índices de diversidad y se aplicaron pruebas de significancia estadística (95\% de significancia) y análisis multivariados (CCA, PCA); el tiempo de muestreo comprendió seis meses. Resultados: A nivel espacial, se encontraron diferencias significativas $(p<0,05)$ en los parámetros físico-químicos (a excepción de los nitritos) y la diversidad; las agrupaciones en el análisis de componentes principales (PCA) y análisis de correspondencia canónica (CCA), identificaron estos cambios espaciales. Con respecto a lo temporal, para lo físico-químico no hubo diferencias significativas ( $p>0,05)$, pero sí en las variables biológicas (a excepción de la densidad); en términos de similitud, el agrupamiento fue independiente del tiempo, donde se identificó asociaciones espaciales mayores a $70 \%$ en los grupos; en la composición, se identificó la dominancia del orden Diptera. Conclusión: Los cambios espaciales en las variables físico-químicas y biológicas parecen ser producto de la influencia de la cabecera municipal de Totoró como tensor antrópico; en lo temporal se puede considerar que la dinámica del río puede tener algún grado de influencia sobre la mayoría de los índices de diversidad, pero no sobre las variables físico-químicas.
\end{abstract}

Palabras clave: Agrupación, Contaminación, Físico-química, Índices de diversidad, Significancia estadística.

\begin{abstract}
Objectives: In the present study the effect of human activity on aquatic macroinvertebrate communities in the Cofre River were analyzed, taking into account the spatial and temporal variation. Methodology: Four study sites were identified based on changes in $\mathrm{pH}$ and conductivity; organisms were collected according to the methodology proposed in this research, together with physic-chemical parameters as supplemental data; data analysis included diversity indices, tests of statistical significance $(95 \%$ level) and multivariate analysis (CCA, PCA); sampling lasted six months. Result: At a spatial level significant differences $(p<0.05)$ were found between the physical-chemical parameters (except nitrites) and diversity; clusters in the principal components analysis (PCA) and canonical correspondence analysis (CCA), identified these spatial changes. regarding the temporal, for physic-chemical no significant differences were found ( $p>0.05$ ), but if for the biological variables (except for the density); in terms of similarity between sampling stations, clustering was independent of the time, where higher spatial associations $70 \%$ was identified in the groups; in the compositions, Diptera order domi-
\end{abstract}

\footnotetext{
Grupo de Estudios en Recursos Hidrobiológicos Continentales, Departamento de Biología, Universidad del Cauca, Popayán, Colombia.e-mail: jhotas24@gmail.com hilldier@gmail.com

2 Grupo de Estudios en Zooplancton Neotropical, Universidad del Cauca, Popayán, Colombia. e-mail: ceandrade2001@ hotmail.com Fecha recepción: Deciembre16, 2013 
nated. Conclusion: the spatial changes in the physicchemical and biological variables seem to be the result of the influence of the municipality of Totoró as anthropogenic pollution impact; in the temporal aspect, the dynamics of the river may have a certain influence on most diversity indices, but not on physic-chemical variables.

Keywords: Contamination, Diversity indices, Group, Physic-chemical, Statistical significance.

\section{Introducción}

Los ecosistemas acuáticos continentales en Colombia se caracterizan por presentar gran variedad de macroinvertebrados acuáticos (Roldán y Ramírez 2008), determinada por las condiciones tropicales en cada uno de los diferentes hábitats presentes en ríos, lagos y embalses. Estas condiciones pueden ser factores bióticos como las interacciones entre especies respecto a la depredación, la competencia y el parasitismo (Molina et al. 2008), que definen el establecimiento de un organismo dentro de la columna del agua.

Además de los factores bióticos, las condiciones físico-químicas son determinantes en el establecimiento de estos organismos; su distribución espacial está influida por el caudal y la velocidad de la corriente (Alvares 2009, Orozco y Rojas 2009); pueden causar cambios en la comunidad las concentraciones de oxígeno disuelto, dióxido de carbono y medidas de conductividad, $\mathrm{pH}$ y temperatura, entre otros parámetros, asociándolos con la contaminación del agua, sobre todo de origen antrópico (Caicedo y $\mathrm{Pa}$ lacio 1998, Giller y Malmqvist 1998, Roldán et al. 2001, Ozcos et al. 2006). Ramírez y Roldán (1989) mencionan que los tensores antrópicos definen variaciones drásticas en las condiciones físico-químicas y biológicas dentro de los cuerpos hídricos

Los macroinvertebrados acuáticos son organismos que responden a las condiciones del medio, por lo que se consideran muy buenos bioindicadores. Resh (2008) y Bonada et al. (2006) mencionan que son los organismos más utilizados con base en ciertos criterios particulares relacionados con su importancia de poseer una amplia distribución geográfica, tener un gran número de especies que generan variedad de respuestas, el muestreo es sencillo y económico, son de carácter sedentario y se pueden de- sarrollar experimentos en el laboratorio. En Colombia, Pinilla (2000) publicó una recopilación bibliográfica sobre la importancia de los macroinvertebrados en el tema bioindicación. Este concepto de bioindicación no solo hay que verlo desde el aspecto del organismo, es importante tener en cuenta la comunidad de macroinvertebrados como conjunto, porque esta identifica de una manera más compleja los niveles de contaminación o alteración en el ecosistema acuático. La manera de poder identificar cambios dentro de la comunidad es a partir de la medición de su diversidad (Moreno 2001).

En el presente trabajo se estudió un tramo de la microcuenca del río Cofre, influido por el casco urbano del municipio de Totoró. El objetivo principal fue interpretar la variación espacial y temporal de la comunidad de macroinvertebrados acuáticos, a partir de los cambios que ejerce el tensor antrópico sobre su composición y diversidad; esta información biológica se contrastó con la variación de algunos parámetros físico-químicos básicos.

\section{Metodología}

Área de estudio. El tramo estudiado en el río Cofre se localiza en un rango altitudinal que va desde los 2.622 metros hasta los 2.420 metros en la cordillera Central, cuenca alta del río Cauca, municipio de Totoró. La temperatura media $14^{\circ} \mathrm{C}$ y la precipitación promedio registrada en los seis meses de campo fue 187,45 mm. La extensión del área urbana es de $92 \mathrm{~km}^{2}$, con aproximadamente 1.233 habitantes. El municipio está ubicado a $30 \mathrm{~km}$ NE del municipio de Popayán. Según Holdridge (1967), la zona del área de estudio corresponde a un un bosque húmedo montano bajo con transición a bosque muy húmedo montano bajo (bh-MB//bmh-MB).

Fase de campo y laboratorio. El tiempo de muestreo abarcó los meses de diciembre 2009 a mayo de 2010, con una salida de campo mensual. Las estaciones de muestreo se fijaron de acuerdo con los cambios registrados en los valores de $\mathrm{pH}$ y conductividad; la primera estación se ubicó antes de

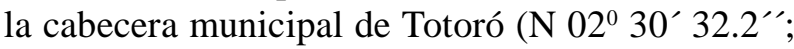
W $076^{0} 23^{\prime} 28.5^{\prime \prime}$ a 2.622 metros de elevación); la segunda, tercera y cuarta estación se ubicaron después de la cabecera municipal, con coordenadas $\mathrm{N}$ $02^{0} 30^{\prime} 33.1^{\prime \prime}$; W $076^{0} 24^{\prime} 33.1^{\prime \prime}$ a 2.517 metros de 
elevación, N $02^{0} 30^{\prime} 40.2^{\prime \prime}$; W $076^{0} 24^{\prime} 56.1^{\prime \prime}$ a 2.494 metros de elevación, $\mathrm{N} 02^{0} 31^{\prime} 28.6^{\prime \prime}$; W $076^{0} 25^{\prime}$ 08.7” a 2.410 metros de elevación (Figura 1).

Para la recolección de los macroinvertebrados acuáticos se utilizó una red surber $\left(0,25 \mathrm{~m}^{2}\right)$ con diámetro de poro de $0,5 \mathrm{~mm}$; se realizaron cuatro replicas que abarcan $1 \mathrm{~m}^{2}$ en cada orilla y centro del sistema lotico. Las muestras se preservaron en recipientes plásticos con alcohol industrial al $95 \%$ y luego se llevaron al laboratorio para conteo e identificación con la ayuda de guías y claves específicas entre ellas (McCafferty 1981, Roldán 1988, Klemm 1995, Epler 1995, Epler 1996, Merritt y Cummins 1996, Merrit et al. 2008, Voshell 2002, Pescador et al. 2002, Pescador et al. 2004, Domínguez et al. 2006, Heckman 2008, Domínguez y Fernández 2009, Springer et al. 2010).

Se utilizó una sonda multiparamétrica YSI 556 MPS para registro de datos in situ de oxígeno disuelto (OD), $\mathrm{pH}$, conductividad y temperatura del agua. Con un espectrofotómetro MERCK SQ118 se determinó nitritos $\left(\mathrm{NO}_{2}\right)$ y amonio $\left(\mathrm{NH}_{4}\right)$. El valor

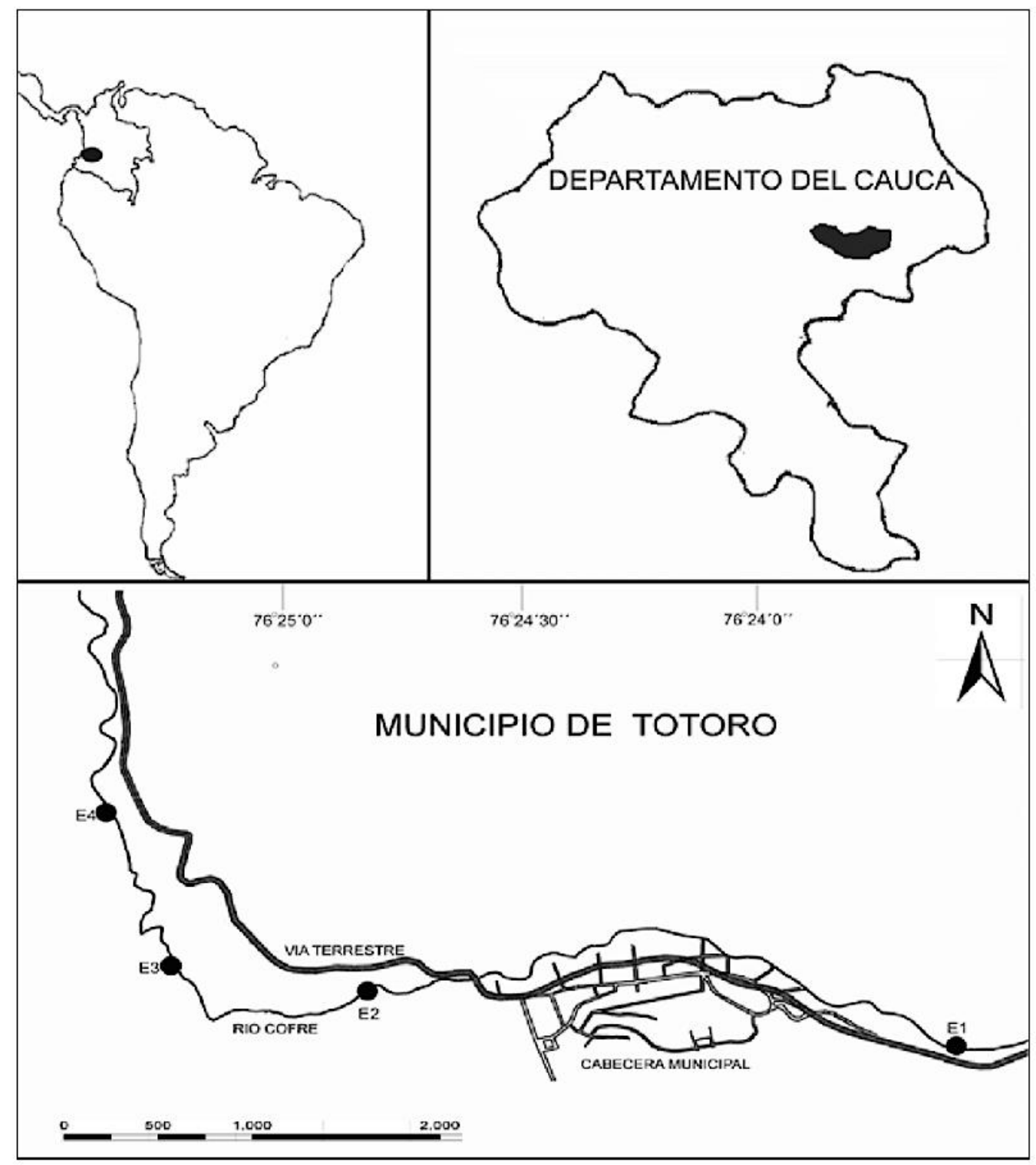

Figura 1. Área de estudio y estaciones de muestreo 
del dióxido de carbono se calculó con el kit de Aquamerck; para hallar el valor de $\mathrm{DBO}_{5}$ se recolectó dos litros de agua por estación y se definió el valor en el laboratorio por el test de cinco días, indicado en el método estándar (Baird 2005).

Análisis de datos. Con el programa SPSS V 11.5 se aplicó la prueba no paramétrica de Kruskal-Wallis (ANOVA no paramétrica donde se mide la tendencia de las muestras asumiendo como hipótesis nula que todas las poblaciones tienen la misma mediana (Guisande et al. 2006), para relacionar las estaciones y meses de muestreo, respecto a los índices de diversidad y variables físico-químicas. Con la implementación del programa Past V. 2.1 se definió la diversidad de Shannon-Weaber $\left(H^{\prime}\right)$, el índice de Pielou $(J)$, la riqueza específica $(S)$, la densidad $(N)$ y el índice de similitud de Bray-Curtis basado en las abundancias de los individuos, para las cuatro estaciones. Utilizando el programa R V.3.0.2 se desarrolló un análisis de correspondencia canónica (CCA) mediante la librería «vegan», donde se relacionó las variables físico-químicas con las estaciones de muestreo y la comunidad de macroinvertebrados acuáticos, teniendo en cuenta la temporalidad; con este mismo software también se desarrolló un análisis de componentes principales (PCA) utilizando la librería «Rcmdr», cuya relación tiene en cuenta las estaciones de muestreo con las variables físico-químicas en la temporalidad.

\section{Resultados}

Aspectos físico-químicos. En la Tabla 1 se ilus- tran los resultados promedios con su respectivo coeficiente de variación de las variables físico-químicas registradas durante el tiempo de estudio. Al aplicar la prueba paramétrica de Kruskal-Wallis se encuentran diferencias significativas $(\mathrm{p}<0,05)$ en las variables físico-químicas (a excepción de los nitritos) con respecto a las estaciones de muestreo (Tabla 2) pero no en los meses de muestreo ( $p>0,05)$ (Tabla 3 ). Este resultado permitió desarrollar un análisis de componentes principales (Figura 2), donde se identifica la relación que existe entre las variables físicoquímicas y cada estación de muestreo. Los primeros dos ejes explicaron el 66,3\% de la varianza. Con el componente 1 en el extremo negativo se sitúan las variables dióxido de carbono $\left(\mathrm{CO}_{2}\right)$, conductividad (Cond), amonio $\left(\mathrm{NH}_{4}\right)$ y demanda biológica de oxígeno $\left(\mathrm{DBO}_{5}\right)$, correlacionadas con la estación dos; en el extremo positivo se asocian las variables oxígeno disuelto (OD) y $\mathrm{pH}$, correlacionadas con las estaciones uno y cuatro. El componente dos explica las variables nitritos $\left(\mathrm{NO}_{2}\right)$ y temperatura, relacionadas con las estaciones tres y cuatro.

\section{Aspecto biológico}

Composición. Se colectaron en total cinco phyla, ocho clases, 15 órdenes, 35 familias, 49 géneros y 5.827 organismos, donde la clase Insecta fue la categoría taxonómica con la mayor densidad de individuos identificados en campo y laboratorio (Anexo 1). A nivel de órdenes (Figura 3), Diptera dominó en número de géneros en las cuatro estaciones de muestreo, seguidos de Coleoptera, Trichoptera y Ephemeroptera. También se puede detallar que existe una mayor riqueza de géneros en la estación uno.

Tabla 1. Estadística descriptiva*

\begin{tabular}{|c|c|c|c|c|c|c|c|c|}
\hline \multirow{2}{*}{ Variable } & \multicolumn{2}{|c|}{ E1 } & \multicolumn{2}{|c|}{ E2 } & \multicolumn{2}{|c|}{ E3 } & \multicolumn{2}{|c|}{ E4 } \\
\hline & $\mathbf{x}$ & CV & $\mathbf{x}$ & CV & $\mathbf{x}$ & CV & $\mathbf{x}$ & CV \\
\hline Temperatura del agua & 12,58 & 8,03 & 14,45 & 5,39 & 15,43 & 6,74 & 16,21 & 7,03 \\
\hline $\mathrm{pH}$ & 7,32 & 2,21 & 6,94 & 2,86 & 7,10 & 2,88 & 7,22 & 2,35 \\
\hline Conductividad (mS/cm) & 42,90 & 31,9 & 79,91 & 12,9 & 57,80 & 37,41 & 39,99 & 28,02 \\
\hline $\mathrm{CO}_{2}(\mathrm{mg})$ & 1,18 & 0,32 & 2,85 & 35,7 & 1,37 & 35,30 & 1,18 & 0,47 \\
\hline $\mathrm{OD}^{2}(\mathrm{mg})$ & 8,53 & 9,62 & 5,75 & 11,9 & 7,55 & 16,67 & 7,91 & 10,67 \\
\hline $\mathrm{DBO}_{5}$ & 1,51 & 18,2 & 2,13 & 10,2 & 1,85 & 11,82 & 1,55 & 17.19 \\
\hline Amonio (mg/l & 0,00 & 0,00 & 0,17 & 152,6 & 0,00 & 244,9 & 0,00 & 0,00 \\
\hline Nitritos (mg/l) & 0,02 & 48,9 & 0,03 & 41,9 & 0,03 & 46,48 & 0,05 & 36,51 \\
\hline
\end{tabular}

* Promedio $(X)$ y coeficientes de variación de Pearson $(\mathrm{CV})$ de las variables fisicoquímicas en las estaciones de muestreo 


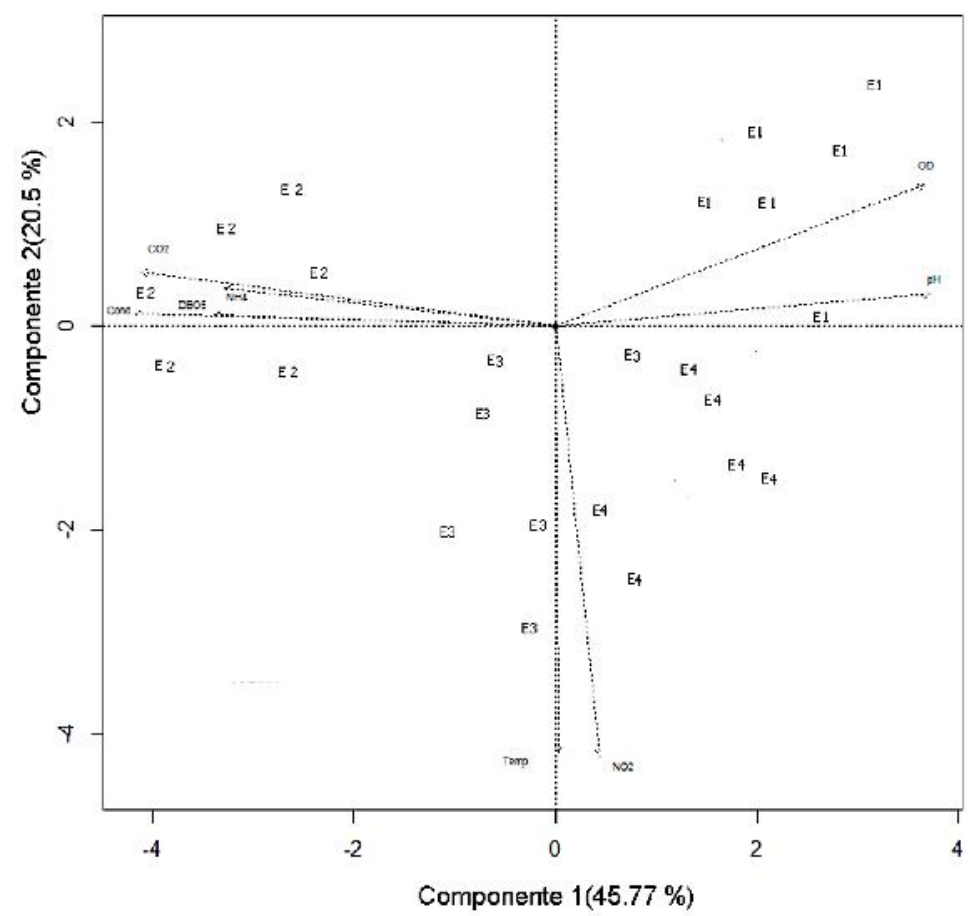

Figura 2. Análisis de componentes principales. Se representa el coeficiente de correlación de cada función definido por cada componente.

Tabla 2. Prueba no paramétrica de Kruskal-Wallis*

\begin{tabular}{lcccccccc}
\hline & Temp. & OD & $\mathbf{C O}_{2}$ & Nitritos & DBO5 & pH & Conduct. & Amonio \\
\hline$\chi^{2}$ & 13,5 & 13,05 & 8,22 & 10,27 & 14,4 & 7,42 & 13,36 & 7,23 \\
gl & 3 & 3 & 3 & 3 & 3 & 3 & 3 & 3 \\
Sig. asintót. & 0,002 & 0,005 & 0,42 & 0,16 & 0,002 & 0,6 & 0,004 & 0,065 \\
\hline
\end{tabular}

* La tabla indica el valor de $x^{2}$, los grados de libertad y el valor de significancia estadística. Variable dependiente «parámetros físico-químicos» y variable independiente «estación». $\mathrm{p}<0,05$

Tabla 3. Prueba no paramétrica de Kruskal-Wallis*

\begin{tabular}{lcccccccc}
\hline & Temp. & OD & CO $_{2}$ & Nitritos & DBO5 & pH & Conduct. Amonio \\
\hline$\chi^{2}$ & 3,456 & 5,272 & 4,94 & 6,74 & 2,499 & 1,77 & 4,133 & 5,726 \\
gl & 5 & 5 & 5 & 5 & 5 & 5 & 5 & 5 \\
Sig. asintót. & 0,509 & 0,423 & 0,42 & 0,24 & 0,777 & 0,88 & 0,53 & 0,334 \\
\hline
\end{tabular}

* La tabla indica el valor de $x^{2}$, los grados de libertad y el valor de significancia estadística. Variable dependiente, parámetros físico-químicos y variable independiente mes. $p>0,05$

Diversidad. Al aplicar la prueba no paramétrica de Kruskal-Wallis sobre los datos calculados para los índices de diversidad alfa (Anexo 2) se encuentran diferencias significativas entre el índice ShannonWeaber $\left(H^{\prime}\right)$, Pielou $\left(J^{\prime}\right)$, riqueza específica $(S)$ y densidad $(N)$, con respecto a las estaciones de muestreo (Tabla 4), al igual que con los meses de muestreo, con excepción en la densidad, que no es significativa (Tabla 5).

Se realizó un clúster de Bray-Curtis (Figura 4), cuyo grado de similitud tiende a agrupar los muestreos de cada sitio; este resultado muestra que la variabilidad espacial es mayor que la temporal. Se observan tres grandes agrupaciones: $\mathrm{X}$, reúne las estaciones 


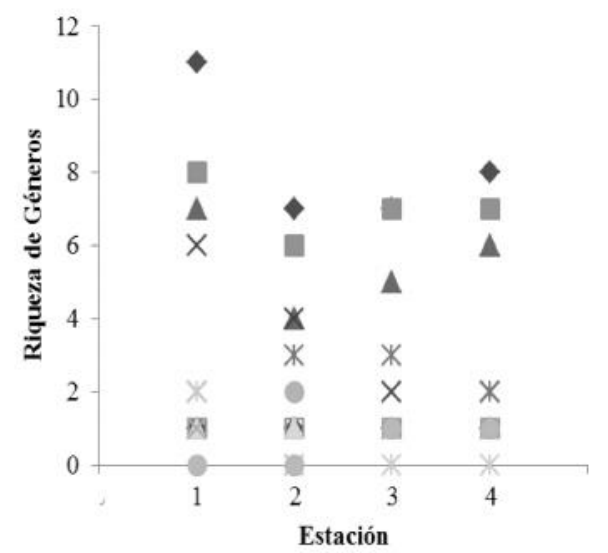

Figura 3. Riqueza de géneros para cada orden de macroinvertebrados acuáticos, presentes en las estaciones.

tres y cuatro con $72 \%$ de similitud, $Y$ la estación uno con $70 \%$ y finalmente $\mathrm{Z}$ la estación dos con $72 \%$.

\section{Análisis de correspondencia} canónica (CCA). Los resultados del análisis de correspondencia canónica (Figura 5) muestran que los dos primeros ejes explican un porcentaje medio de $54,8 \%$ de la varianza total. El primer eje canónico se asocia positivamente con la conductividad, $\mathrm{DBO}^{5}$ y $\mathrm{CO}_{2}$, relacionándose con la estación dos y los taxones Planorbiidae (Plano), Lombriculidae (Lomb), Physa sp. (Phy), Chironomidae (Chiro) y Tubifex sp. (Tub); por el lado negativo, se asocia con el oxígeno disuelto (OD) y $\mathrm{pH}$, correlacionado con la estación uno y ubicándose los taxones Ochrotrichia sp. (Ochro), Chordodidae (Nema), Elodes sp. (Elod), Lymnessia sp. (Lymn), Contulma sp. (Cotu), Phanocerus sp. (Pha) y Limonicola sp. (Lim). Con el eje dos se relaciona negativamente las variables temperatura (Temp) y nitritos $\left(\mathrm{NO}_{2}\right)$, asociadas con las estaciones tres y cuatro, y a los taxones Hetaerina (Het) y Atanatolica (Atan).
- Diptera

Coleoptera

$\Delta$ Trichoptera

X Ephemeroptera

* Basommatophora

- Lepidoptera

+ Amphipoda

- Veneroida

- Haplotaxida

- Lombriculida

Glossiphoniiformes

Tricladia

Gordoidea

Acari

- Odonata
Tabla 4. Prueba no paramétrica de Kruskal-Wallis*

\begin{tabular}{lcccc}
\hline & $\mathbf{N}$ & $\mathbf{S}$ & $\mathbf{H}^{\prime}$ & $\mathbf{J}^{\prime}$ \\
\hline$\chi^{2}$ & 81,264 & 31,192 & 50,929 & 85,146 \\
gl & 3 & 3 & 3 & 3 \\
Sig. asintót. & 0,001 & 0,001 & 0,001 & 0,001 \\
\hline
\end{tabular}

* La tabla indica el valor de $x^{2}$, los grados de libertad $y$ el valor de significancia estadística. Variable dependiente: índices de diversidad (densidad $(N)$, riqueza específica $(S)$, índice de Shanon-Weaber $(H)$, índice de Pielou $(J)$ y variable independiente: estación. $p<0,05$

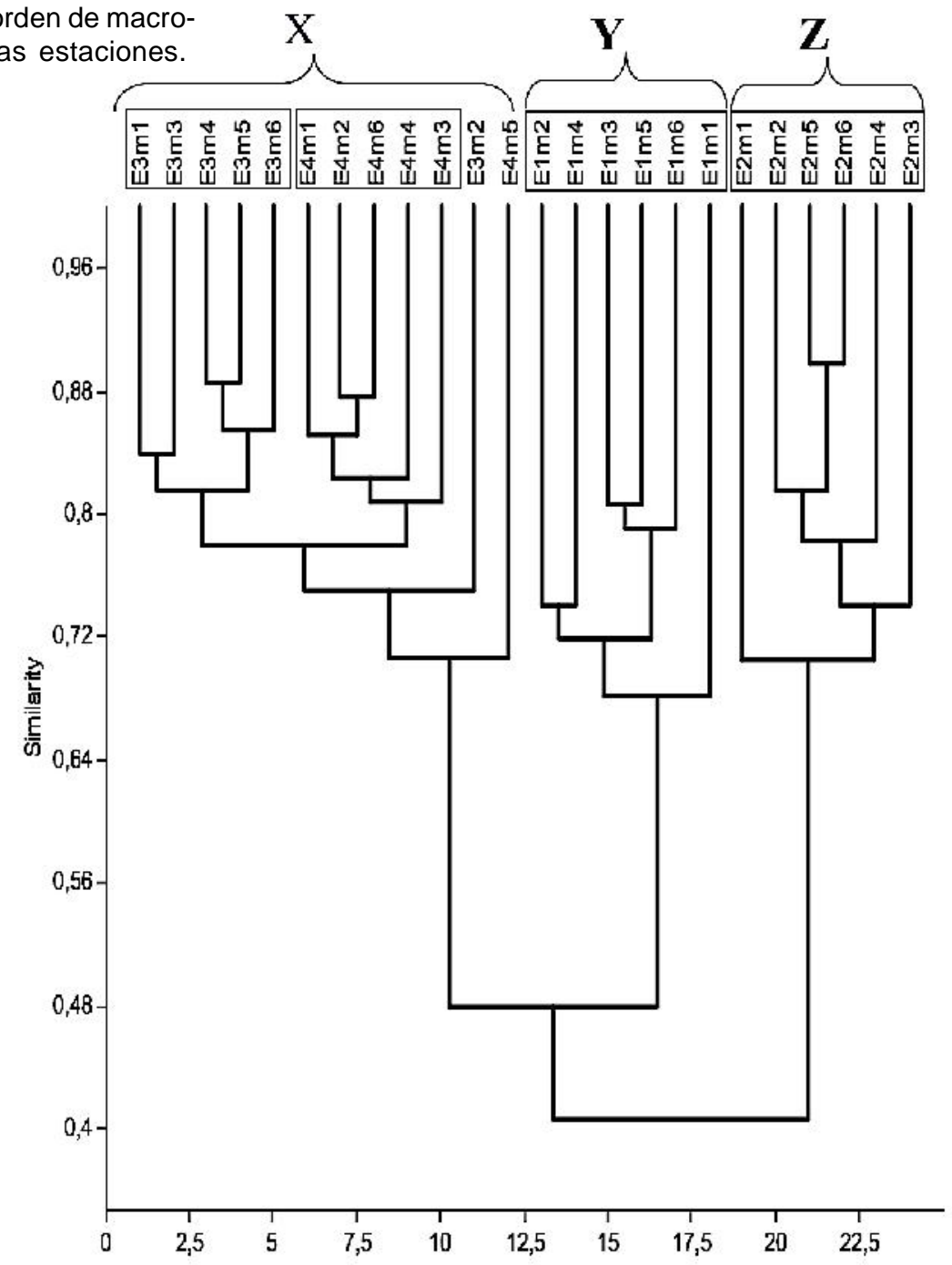

Figura 4. Dendrograma de similitud con base en el índice de Bray-Curtis

Se identifican tres grupos conformados por la estación 1 (X) que es la menos intervenida, las estaciones 3 y $4(\mathrm{Y})$ en proceso de recuperación y la estación 2 (Z) donde se ubica el tensor antrópico. E1 a E4: Estaciones 1 a 4; $\mathrm{m} 1$ a m6: meses de muestreo. 


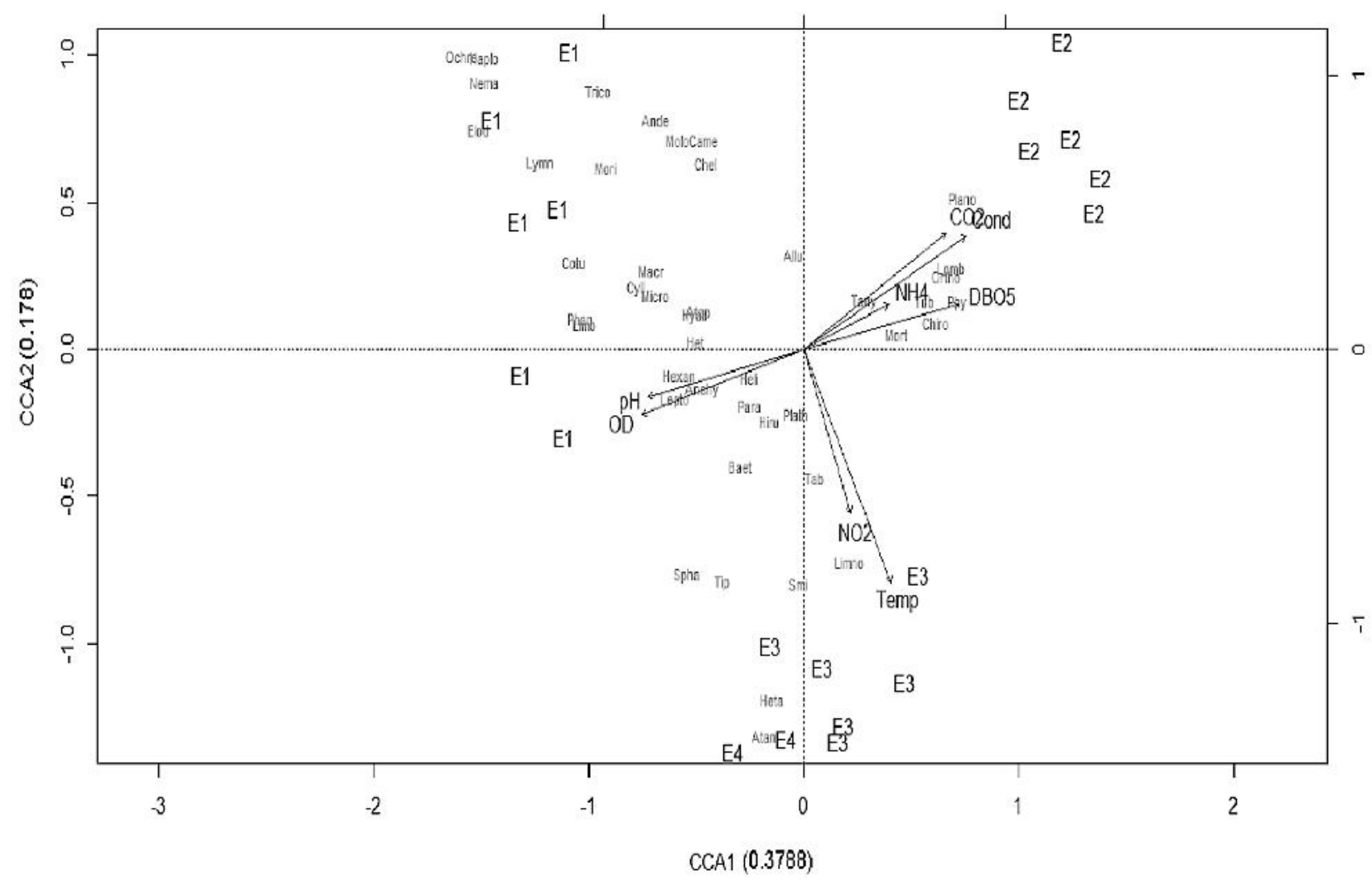

Tabla 5. Prueba no paramétrica de Kruskal-Wallis*

\begin{tabular}{lcccc}
\hline & $\mathbf{N}$ & $\mathbf{S}$ & $\mathbf{H}^{\prime}$ & $\mathbf{J}^{\prime}$ \\
\hline$\chi^{2}$ & 5,059 & 14,202 & 17,289 & 15,682 \\
gl & 5 & 5 & 5 & 5 \\
Sig. asintót. & 0,409 & 0,014 & 0,004 & 0,008 \\
\hline
\end{tabular}

* La tabla indica el valor de $x^{2}$, los grados de libertad $y$ el valor de significancia estadística. Variable dependiente «índices de diversidad (densidad $(N)$, riqueza específica $(S)$, índice de Shanon-Weaber $(H)$, índice de Pielou (J)»y variable independiente «mes». $p<0,05$.

\section{Discusión}

Los cambios en las variables físico-químicas no se ven afectados significativamente por la temporalidad, debido a una posible estabilidad de la dinámica del río durante el tiempo de muestreo. En cambio, a nivel espacial si se observan estas diferencias, específicamente en la estación dos, al existir un incremento de los valores registrados para $\mathrm{DBO}_{5}$, conductividad, amonio y $\mathrm{CO}_{2}$ que indican contaminación por aporte alóctono de materia orgánica, al igual que la disminución del oxígeno disuelto y el $\mathrm{pH}$, cuyos valores están más correlacionados con las estaciones uno y cuatro, con mejores condiciones de calidad del agua. En esta esta- ción se encuentra la zona de vertimientos de aguas servidas domésticas, alcantarillado, residuos hospitalarios y matadero, lo que ocasiona un cambio en el sustrato al observar una mayor acumulación de lodo que cubre los diferentes componentes del lecho (arena, bloques, cantos rodados, etc.). Según Tank et al. (2010) esta acumulación hace parte del proceso de retención por parte de los sustratos y el componente biológico principalmente de características bénticas, lo que puede definir la presencia o ausencia de algún organismo (Reice 1980) y las condiciones del hábitat (Prat et al. 2009).

Volgaropoulos et al. (1987), mencionan que el oxígeno disuelto y el $\mathrm{pH}$ son buenos indicadores de contaminación por materia orgánica al disminuir su concentración; al igual, un aumento en la concentración de $\mathrm{CO}_{2}, \mathrm{DBO}_{5}$, conductividad, $\mathrm{CO}_{2}$, amonio y nitritos, puede indicar alteración de la masa del agua como lo estipula Marín (2006). Estos cambios influyen en la dinámica de la comunidad de macroinvertebrados, al generar nuevas condiciones de adaptabilidad dentro del sistema acuático, lo que define la presencia de organismos sensibles 
o tolerantes a la contaminación orgánica (AlbaTercedor 1996, Vivas et al. 2002). Zamora (1998) y Feriz y Ortega (2009) en el municipio de Popayán, y por Villareal (2007) en el municipio de Sibundoy, encontraron una disminución en la diversidad de macroinvertebrados por cambios en las condiciones físico-químicas, que favorecen el desarrollo de organismo tolerantes de la familia Chironomidae y del género Tubifex.

Los nitritos son indicadores de contaminación de características fecales (Tchobanoglous y Burton 1991). Su aumento de concentración a lo largo del gradiente ambiental se debe a que posiblemente existe una acumulación de este nutriente por la influencia que tienen las aguas residuales y una mayor presencia de ganadería aguas abajo del vertimiento, que vierten material fecal. En teoría, la presencia de nitritos en aguas superficiales es muy baja con valores menores a 0,1 mg/l (Marín 1995), lo que indicaría que su concentración en el río $(0,03 \mathrm{mg} / \mathrm{l}$ en promedio) no alteraría significativamente sus condiciones normales.

El aumento en los valores de temperatura del agua se debe sobre todo a la disminución altitudinal entre los puntos de muestreo. Machado y Roldán (1981), consideran que los cambios en la altura al nivel del mar es un factor determinante sobre esta variable ambiental. Cabe destacar, que la hora de registro puede influir en este parámetro, debido a su relación con la incidencia solar diaria, que puede aumentar o disminuir la temperatura de la columna del agua.

La mayor abundancia de Diptera se debe a su amplia distribución y adaptabilidad a diferentes condiciones ambientales. De acuerdo con Zúñiga (1985), Roldán (1988), Escobar (1989) y Mora y Soler (1993), este orden ocupa medios con aguas limpias bien oxigenadas, como el caso de la familia Blephariceridae (Género Limonicola encontrado en las estaciones uno y cuatro) y aguas contaminadas, como la familia Chironomidae, colectados en las cuatro estaciones. Si se tiene en cuenta el BMWP/Col (Zamora 2007), las familias de Dipteros van desde la puntuación 10 hasta la dos, lo que evidencia su amplio rango de distribución en los ecosistemas acuáticos con diferentes niveles de perturbación.

Roldán (1988) considera que la mayor riqueza para los órdenes Coleoptera y Trichoptera se evidencia en sistemas lóticos fríos, característica impor- tante del cuerpo hídrico estudiado. El orden Coleoptera es el grupo más numeroso de organismos conocidos (Lawrence y Newton 1995) y aunque la mayoría son terrestre (Bouchard 2004), se logra encontrar algunos grupos representantes en el medio acuático (White y Roughley 2008). Estos organismos han tenido reconocimiento en términos de bioindicación (Bournaud et al. 1992), de ahí su importancia al momento de evaluar un sistema en términos ecológicos; por ejemplo, los taxones hallados en el presente estudio pertenecen a las familias Ptilodactylidae y Scirtidae que indica aguas de muy buena calidad (Zamora 2007) y la familia Elmidae indicadores de aguas intermedias (Zamora 2007). Para el orden Trichoptera, la utilidad que se da en estados larvales determina la gran diversidad ecológica en medios acuáticos (Mackay y Wiggins 1979), por tal razón se puede encontrar un sin número de adaptaciones definidas por el tipo de sustrato que habiten (Springer 2010). En el presente estudio, este orden fue encontrado en arena, gravas, rocas y vegetación ribereña. Por último, está el orden Ephemeroptera, cuyos individuos se encuentran en aguas bien oxigenadas y con contaminación moderada (Roldán 1988). Se registra su mayor riqueza en un rango altitudinal entre 1000-2000 metros (Escobar 1989). Zamora (2000) y Feriz y Ortega (2009) encontraron una dominancia taxonómica similar a la de este estudio, lo que permite inferir que a nivel regional, este tipo de organización en las dominancias dentro de la comunidad de macroinvertebrados podría ser muy común en los ecosistemas acuáticos andinos del Cauca.

Existe una alta similitud interna en cada agrupación del cluster, lo que sustenta que no existan diferencias significativas en términos de densidad en los meses de muestreo; si el análisis se enfoca entre agrupaciones, se puede observar el efecto que tiene la influencia del tensor antrópico sobre la comunidad, encontrando una asociación del $48 \%$ entre X y Y y estos dos grupos respecto a $\mathrm{Z}$ del $42 \%$. En relación con los índices de diversidad, se evidencian cambios significativos en el aspecto espacial por la influencia del tensor antrópico como se ha discutido antes y en lo temporal por la influencia que puede tener la dinámica del río sobre la comunidad. Se puede intuir que la dinámica puede tener un comportamiento de variable selectiva que influye en la riqueza, equidad y dominancia, pero no sobre la densidad de organis- 
mos como lo demuestra los resultados en el clúster de Bray Curtis (basado en la densidad) y la prueba no paramétrica. Esta idea intuitiva se puede apoyar con los trabajos de Posada et al. (2008) y Longo et al. (2010), quienes analizan la influencia de la temporalidad y variables físicas sobre el establecimiento de la comunidad de macroinvertebrados acuáticos, identificando agrupaciones de organismos que se establecen en los hábitats de acuerdo con sus estructuras adaptativas.

Los taxones agrupados positivamente con el primer eje son organismos indicadores de contaminación o condiciones de anoxia (Machado y Roldán 1981, Zúñiga 1985, Roldán 1988, Escobar 1989, Mora y Soler 1993, Muñoz y Prat 1996) que se generan al existir una alteración del ambiente acuático por parte de la cabecera municipal de Totoró y privilegia el desarrollo de sus poblaciones, como por ejemplo, el aumento de lodo o material particulado fino en el sustrato, sobre todo la familia Chironomidae y los géneros Tubifex sp. y Physa sp. Para el eje 1 negativo, se hallaron en la estación 1 organismos indicadores de buena calidad (Roldán 1988, Escobar 1989, Ramírez y Roldán 1989, Zamora y Alba 1996), tales como los géneros Limonica sp. y Limnesya sp. Con el eje 2 se asocian organismos que pueden vivir en ambientes acuáticos con contaminación moderada a limpia (Machado y Roldán 1981, Escobar 1989, Ramírez y Roldán 1989).

\section{Conclusiones}

A nivel espacial, los parámetros físico-químicos (a excepción de los nitritos) y la diversidad presentaron diferencias significativas, asociados con el tensor antrópico. En cambio, en el aspecto temporal, no hubo diferencias significativas para las variables abióticas, pero sí en las biológicas (a excepción de la densidad).

Un aumento en los valores de las variables físico-químicas $\mathrm{DBO}_{5}$, conductividad, amonio, $\mathrm{CO}_{2}, \mathrm{y}$ disminución en el $\mathrm{pH}$ y oxígeno disuelto en la estación 2 , indican contaminación por aporte alóctono de aguas residuales provenientes de la cabecera municipal de Totoró. La acumulación de lodo sobre los sustratos presentes en los sitios de estudio, también son un indicio de acumulación de materia orgánica que beneficia a la fauna de características bénticas y tolerantes con la contaminación.

Los nitritos son la única variable que aumenta su concentración a lo largo del gradiente ambiental; este se debe a un efecto continuo de aguas residuales y una mayor presencia de ganadería. Al igual, la temperatura también aumenta sus valores, determinado por la relación que tiene con la altitud y la hora de muestreo en el día.

Las condiciones físico-químicas y la influencia de la cabecera municipal sobre el río Cofre, definen los taxones macroinvertebrados acuáticos establecidos en cada estación de muestreo, lo que generó un cambio en la dinámica de la comunidad en términos de composición y diversidad.

\section{Agradecimiento}

A la Universidad del Cauca y al Grupo de Estudios en Recursos Hidrobiológicos Continentales (GERHC), quienes apoyaron el desarrollo del proyecto con el préstamo de equipos, laboratorio y bibliografía.

\section{Literatura citada}

Alba-Tercedor J. 1996. Macroinvertebrados acuáticos y calidad de las aguas de los ríos. IV simposio del Agua en Andalucía (SIAGA), España.

Alvares M. 2009. Estudio de la variabilidad espacio-temporal de las comunidades de macroinvertebrados bentónicos en los ecosistemas fluviales de Catambria. Repercusiones para la aplicación de la directiva marco del agua. (Tesis doctoral). España: Universidad de Catambria. 177 p.

Baird RB. 2005. Biochemical Oxygen Demand (BOD). In: Standard methods for the examination of water $y$ wastewater. $21^{\text {st }}$ ed. Eaton DA, Clesceri LS, Rice EW, Greenberg AE (eds). Parte 5000, 5-2 a 5-7. American Public Health Association.

Bonada N, Prat C, Resh VH, Statzner B. 2006. Develoments in aquatic insects biomonitoring: A comparative analisys of recent approaches. Annu Rev Entomol. 51: 495-523.

Bouchard RW Jr. 2004. Guide to aquatic invertebrates of the upper midwest. Identification manual for students, citizen monitors, and aquatic resource professionals. St. Paul: Water Resources Center, University of Minnesota; 207 $\mathrm{p}$.

Bournaud M, Richoux P, Usseglio-Polatera P. 1992. An approach to the synthesis of qualitative ecological information from aquatic Coleoptera communities. Reg Rivers: Res \& Management. 7: 165-80.

Caicedo O, Palacio J. 1998. Los macroinvertebrados bentónicos y la contaminación orgánica en la quebrada La Mosca (Guanare, Antioquia, Colombia). Actualid Biol. 20 (69): 
61-73.

Domínguez E, Molineri C, Pescador ML, Hubbard MD, Nieto C. 2006. Aquatic biodiversity in Latin America. Ephemeroptera of South America. Sofia-Moscow: PENSOFT Publishers; 646 pp.

Domínguez E, Fernández HR. 2009. Macroinvertebrados bentónicos sudamericanos. Sistemática y biología. Tucuman: Fundación Miguel Lilio; 654 pp.

Epler JH. 1995. Identification manual for the larval Chironomidae (Diptera) of Florida. Tallahasee: Department of Environmental Protection Division of Water Facilities; 320 pp.

Epler JH. 1996. Identification manual of the water beetles of Florida (Coleoptera: Dryopidae, Dysticidae, Elmidae, Gyrinidae, Haliplidae, Hydraenidae, Hydrophilidae, Noteridae, Psephenidae, Ptilodactylidae, Scirtidae). Tallahassee: Departament of Environmental Protection Division of Water Facilities; 259 pp.

Escobar A. 1989. Estudio de las comunidades de macrobentónicas en el río Manzanares y sus principales afluentes y su relación con la calidad del agua. Actualid Biol: 18 (65): 45-60.

Feriz D, Ortega Y. 2009. Determinación de la calidad del agua de la quebrada La Cantera, empleando comunidades de macroinvertebrados y parámetros físico-químicos como indicadores. (Trabajo de pregrado). Popayán: Facultad de Ciencias Naturales, Exactas y de la Educación, Universidad del Cauca. 120 pp.

Giller PS, Malmqvist B. 1998. The biology of streams and rivers. Oxford: Oxford University Press; 296 pp.

Guisande C, Barreiro A, Maneiro I, Riveiro I, Vergara A, Vaamonde A. 2006. Tratamiento de datos. Madrid: Ed. Diaz de Santos; 356 pp.

Heckman WC. 2008. Encyclopedia of South America aquatic insects: Odonata-Zigoptera. Ilustrated keys to know families, genera and species in South America. Olympia: Springer; $688 \mathrm{pp}$.

Holdridge LR. 1967. Life zone ecology. San José: Tropical Science Center, IICA; 1982.

Klemm DJ. 1995. Identification guide to the freshwater leeches (Annelida: Hirudiena) of Florida and other southern state. Tallahasee: Department of Environmental Protection, Division of Water Facilities; 82 pp.

Lawrence JF, Newton AF. 1995. Families and subfamilies of Coleoptera (with selected genera, notes, references and data on family-groups names). In: Biology, phylogeny, and classification of Coleoptera. Papers Celebrating the 80th Birthday of Roy A. Crowson. Pakaluk J, Slipinski SA (eds.). Warszawa: Muzeum i Instytut Zoologii PAN; p. 779-1006.

Longo-S M, Zamora-G H, Guisande-G C, Ramírez-R J. 2010. Dinámica de la comunidad de macroinvertebrados en la quebrada Potrerillos (Colombia): Respuesta a los cambios estacionales de caudal. Limnetica. 29 (2): 195-210.

Machado T, Roldán G. 1981. Estudio de las características físicoquímicas y biológicas del río Anorí y sus princi-pales afluentes. Actualid Biol. 10 (35): 3-19.

Mackay RJ, Wiggins GB. 1979. Ecological diversity in
Trichoptera . Ann Rev Entomol. 24: 185-208.

Marín R. 1995. Análisis de aguas y ensayos de tratamiento: principios y aplicaciones. Barcelona: Ed. GPE S.A; 719 pp.

Marín R. 2006. Fisicoquímica y microbiología de los medios acuáticos: Tratamiento y control de calidad del agua. Madrid: Ediciones Díaz de Santos; 336 pp.

McCafferty WP. 1981. Aquatic entomology the fishermen's and ecologist's illustrated guide to insect and their relatives. Boston: Science Books International; 448 pp.

Merrit RW, Cummins KW. 1996. An introduction to the aquatic insects of North America. $3^{\text {rd }}$ ed. Kendall: Hunt Publishing Company; $862 \mathrm{pp}$.

Merrit RW, Cummins KW, Berg MB. 2008. An introduction to the aquatic insects of North America. $4^{\text {th }}$ ed. Kendall: Hunt Publishing Company; 1158 pp.

Molina C, Francois MG, Pinto J, Rosales C. 2008. Estructura de macroinvertebrados acuáticos en un río altoandino de la cordillera real, Bolivia: variación anual y longitu-dinal en relación a factores ambientales. Ecol Aplic. 7: 1-2.

Mora A, Soler S. 1993. Estudio limnológico, con énfasis en los macroinvertebrados bentónicos de la parte alta del río Bogotá (Quebrada del Valle, Planta de tratamiento de Tibitó). (Tesis Biólogo). Bogotá: Facultad de Ciencias, Departamento de Biología, Universidad Nacional de Colombia.

Moreno CE. 2001. Métodos para medir la biodiversidad. Zaragoza: MyT-Manuales y Tesis SEA.1; 83.

Muñoz I, Prat N. 1996. Effects of water abstraction and pollution on macroinvertebrate community in medite-rraneam river. Limnetica. 12 (1): 9-16.

Orozco A, Rojas F. 2009. Estudio de la influencia de algunos factores ecohidráulicos sobre la distribución de los macroinvertebrados acuáticos en un tramo del río Palace. (Trabajo de grado). Popayán: Facultad de Ciencias Naturales, Exactas y de la Educación, Universidad del Cauca. 135 pp.

Ozcos J, Campos F, Escala MC. 2006. Variación de la comunidad de macroinvertebrados bentónicos en relación con la calidad del agua. Limnetica. 25 (3): 683-92.

Prat N, Ríos B, Acosta R, Rieradevall M. 2009. Los macroinvertebrados como indicadores de la calidad de las aguas. En: Macroinvertebrados bentónicos sudame-ricanos. Sistemática y biología. Domínguez E, Fernández HR (ed.). Tucumán: Fundación Miguel Lillo. p. 631-51.

Pescador ML, Rasmussen AK, Harris SC. 2002. Identification manual for the Caddislfly (Trichoptera) Larvae of Florida. Tallahasee: Department of Environmental Protection Division of Water Resource Management; $136 \mathrm{pp}$.

Pescador ML, Barton AR. 2004. Guide to the Mayfly (Ephemeroptera) Nymphs of Florida. Tallahasee: Department of Environmental Protection Division of Water Resource Management; 168 pp.

Pinilla A. 2000. Indicadores biológicos en ecosistemas acuáticos continentales de Colombia. Santa Fe de Bogotá: Universidad Jorge Tadeo Lozano; 67 pp.

Posada-García J, Abril-Ramírez G, Parra-Sánchez L. 2008. Diversidad de los macroinvertebrados acuáticos del Páramo 
de Frontino (Antioquia, Colombia). Caldasia. 30 (2): 44155.

Ramírez J, Roldán G. 1989. Contribución al conocimiento limnológico y de los macroinvertebrados acuáticos de algunos ríos de la región del Urabá antioqueño. Actualid Biol. 18 (66): 113-21.

Reice SR. 1980. The role of substratum in benthic macroinvertebrate microdistribution and litter descomposition in a woodland stream. Ecology. 61 (3): 580-90.

Resh V. 2008. Which group is best? Atributes of different biological assemblages used in freshwater biomonitoring programs. Environ Monit Asses. 138: 131-6.

Roldán G. 1988. Guía para el estudio de los macroinvertebrados acuáticos del departamento de Antioquia. Bogotá: Editorial Presencia Ltda; 217 pp.

Roldán G, Posada J, Gutiérrez J. 2001. Estudio limnológico de los recursos hídricos del parque de Piedras Blancas. Colección Jorge Álvarez Lleras N 18; Bogotá: Academia Colombiana de Ciencias Exactas, Físicas y Naturales; 152 $\mathrm{pp}$

Roldán G, Ramírez J. 2008. Fundamentos de lignología neotropical. $2^{\text {a }}$ ed. Medellín: Editorial Universidad de Antioquia; $440 \mathrm{pp}$.

Springer M. 2010. Trichoptera. Capítulo 7. Macroinvertebrados de agua dulce de Costa Rica I. Rev Biol Trop. 58:151-98.

Springer M, Ramírez S, Hanson P. 2010. Macroinvertebrados de agua dulce de Costa Rica I. Rev Biol Trop. 58. 240 pp.

Tank LJ, Rosi-Marshall EJ, Griffiths NA, Entrekyn SA, Stephen ML. 2010. A review of allochthonous organic matters dinamycs and metabolism in stream. J NAm Benthol Soc. $29(1): 118-46$.

Tchobanoglous G, Burton F. 1991. Wastewater engineering: Treatment, disposal and reuse. $3^{\text {rd }}$ ed. New York: McGrawHill; 920 p.

Villareal L. 2007. Efectos de los vertimientos de aguas residuales domésticas en la calidad biológica y físico-química de la quebrada Lavapies, municipio de Sibundoy, Putumayo.
(Proyecto de grado). Popayán: Facultad de Ciencias Naturales, Exactas y de la Educación, Universidad del Cauca. 98 pp.

Vivas S, Casas J, Pardo I, Robles R, Bonada N, Mellado A. 2002, et al. Aproximación multivariante en la exploración de la tolerancia ambiental de las familias de macroinvertebrados de los ríos del mediterráneo del proyecto GUADALMED. Limnetica. 21 (3-4): 149-73.

Voshell JR. 2002. A guide to common freshwater invertebrates of North America. Blacksburg: The McDonald y Woodward Publishing Company; 442 pp.

Voulgaropoulus A, Fytianos KA, Gounaridoux X. 1987. Correlation of some organic pollution factor in water systems in Northern Greece. WatRes. 21 (3): 253-6.

White DS, Rougley RE. 2008. Aquatic Coleoptera. Chapter 20. In: introduction to the aquatic insects of North America. $4^{\text {th }}$ ed. Merritt RW, Cummins KW, Berg MB (eds.). Kendall: Hunt publishing Company. p. 571-671.

Zamora H. 1998. Niveles de alteración de las comunidades de macroinvertebrados acuáticos del río Molino por efectos de las actividades antrópicas y la contaminación doméstica. Unicauca Cien. 3: 11-30.

Zamora H. 2000. Análisis biogeográfico de los macroinvertebrados acuáticos epicontinentales (MAE) en el departamento del Cauca. Rev Asoc Colomb Cien Biol. 14 (1): 37-64

Zamora H. 2007. El índice BMWP y la evaluación biológica de la calidad del agua en los ecosistemas acuáticos epicontinentales naturales de Colombia. Asoc Colomb Cien Biol. 19: 73-81.

Zamora-Muñoz C, Alba-Tercedor J. 1996. Bioassessment of organically polluted Spanish rivers, using a biotic index and multivariate methods. J N Am Benthol Soc. 15 (3): 332-52.

Zúñiga de CMC. 1985. Estudio de la ecología del río Cali con énfasis en su fauna bentónica como indicador biológico de calidad. Rev AINSA. 8: 63-85. 